\title{
Interpretation of the CALET Electron+Positron Spectrum concerning Dark Matter Signatures
}

\author{
Holger Motz* \\ Global Center for Science and Engineering, Faculty of Science and Engineering, \\ Waseda University, 3-4-1 Okubo, Shinjuku, Tokyo 169-8555, Japan \\ E-mail: motzeaoni.waseda.jp
}

\section{Yoichi Asaoka}

Waseda Research Institute for Science and Engineering, Faculty of Science and Engineering, Waseda University, 3-4-1 Okubo, Shinjuku, Tokyo 169-8555, Japan

E-mail: yoichi.asaoka@aoni.waseda.jp

\section{Saptashwa Bhattacharyya}

${ }^{b}$ Graduate School of Advanced Science and Engineering, Faculty of Science and Engineering, Waseda University, 3-4-1 Okubo, Shinjuku, Tokyo 169-8555, Japan

E-mail: saptashwaberuri.waseda.jp

CALET (CALorimetric Electron Telescope) is in operation on the ISS since October 2015 and directly measures the electron+positron cosmic-ray spectrum up into the TeV-region with fine energy resolution and good proton rejection. Interpretations of the latest results published in [O. Adriani et al. PRL 120, 261102] regarding Dark Matter signatures are presented. Limits on annihilation and decay of Dark Matter were calculated based on an analytic parametrization of the local electron and positron spectra, including a term representing the flux from nearby pulsars as the extra electron-positron-pair source responsible for the positron excess, which is fitted to CALET data and positron flux/fraction data of AMS-02. The expected flux from Dark Matter is calculated with PYTHIA and DRAGON, and added to the parametrization with increasing scale factor until reaching 95\% CL exclusion, returning a limit on the annihilation cross-section or lifetime. By treating systematic uncertainties with known energy dependence as corrections to the fit function, limits were improved compared to all-random errors. Structures appear in the spectrum, which have been investigated as potential Dark Matter signatures by looking for an improvement of the fit quality with addition of flux from Dark Matter. Thereby, annihilation of $350 \mathrm{GeV}$ or decay of $700 \mathrm{GeV}$ Dark Matter to electron-positron pairs is identified as a possible explanation of a step-like structure around $350 \mathrm{GeV}$. The significance of this signature, Dark Matter explanations of other spectral features and possible astrophysical alternatives are discussed

36th International Cosmic Ray Conference -ICRC2019-

July 24th - August 1st, 2019

Madison, WI, U.S.A.

\footnotetext{
* Speaker.
} 


\section{Introduction}

Search for potential Dark Matter signatures in the electron+positron cosmic-ray spectrum is an important mission goal of the CALET project [1], for which it is well suited due to its high energy resolution, promising strong constraints on Dark Matter properties [2]. Dark Matter search in the high-energy electron+positron spectrum is distinguished from indirect Dark Matter search with $\gamma$-rays by the target region being the galactic halo within the short propagation distance of high energy electrons, instead of distant over-dense regions such as the Galactic Center or dwarf galaxies. Therefore it is not subject to effects from the large uncertainty of halo models [3]. On the other hand, indirect Dark Matter search in electron and positron cosmic rays requires a detailed model of the astrophysical background, including possible sources of the positron excess. Thus, the CALET electron+positron spectrum up to $4.8 \mathrm{TeV}$ [4] is combined with the AMS-02 positrononly spectrum up to $1 \mathrm{TeV}$ [5] for this study, which follows the concept explained in Ref. [2] for calculation of limits on Dark Matter annihilation and decay, with a refined background modeling, based on which also structures in the CALET spectrum are interpreted.

\section{Modeling of Electron and Positron Spectra}

To model the positron and electron spectra, different strategies are used for each component, based on what can be inferred from observation and theory. The electron spectrum is expressed as

$$
\Phi_{e}^{-}=C_{e} E^{-\left(\gamma_{e}-\Delta \gamma_{e}\right)}\left(1+\left(\frac{E}{E_{B}}\right)^{\frac{\Delta \gamma_{e}}{s}}\right)^{s} e^{-\left(\frac{E}{E_{\text {cut }}}\right)}+\frac{C_{s}}{C_{\text {norm }}} \Phi_{s\left(e^{-}\right)}+\Phi_{e x},
$$

and the positron spectrum as

$$
\Phi_{e}^{+}=\frac{C_{s}}{C_{n o r m}} \Phi_{s\left(e^{+}\right)}+\Phi_{e x}
$$

Due to their large energy loss in propagation, the spectrum of primary electrons depends on the distribution of individual supernova remnants (SNR) in the galactic neighborhood of the solar system, which is yet mostly unknown. As an effective model of the local (after propagation) primary electron spectrum from all contributing SNR, it is parametrized by a power law with a soft spectral break (index $\gamma_{e}$, break position $E_{b}$, index change $\Delta \gamma_{e}$, softness $s$ ) at low energy and a high-energy exponential cut-off at $E_{\text {cut }_{d}}$ representing radiative energy loss of high energy electrons. Though the measurements $[6,4,7]$ support a cut-off or spectral index change around one TeV, they provide little constraint on the value of $E_{\text {cut }}$ within a range of about $2 \mathrm{TeV}$ to $10 \mathrm{TeV}$, which is therefore treated as a fixed nuisance parameter with a default value of $4 \mathrm{TeV}$.

The secondary positron $\left(\Phi_{s\left(e^{+}\right)}\right)$and electron $\left(\Phi_{s\left(e^{-}\right)}\right)$fluxes are taken from the output of numerical propagation calculation with DRAGON [8] for the nuclei spectra up to Carbon, which is used to establish propagation conditions used consistently from calculation of fluxes from secondaries, pulsars and Dark Matter. With an initial scale factor $C_{\text {norm }}$ obtained from normalizing the proton flux to measurements of AMS-02 [9], a common rescaling factor $\left(C_{s} / C_{n o r m}\right)$ is included in the fit as free parameter to account for uncertainties in secondary production. 
The chosen propagation conditions (also used in [10]) comprise a gradual change in the slope of the diffusion coefficient according to

$$
D(r)=D_{0}\left(\frac{R}{R_{0}}\right)^{\delta_{l}}\left(1+\left(\frac{R}{R_{b}}\right)^{\frac{\delta_{l}-\delta_{h}}{s}}\right)^{-s}
$$

with $\delta_{l}=0.6, \delta_{h}=0.33, R_{0}=4 \mathrm{GV}, R_{b}=300 \mathrm{GV}, D_{0}=1.3 \cdot 10^{28} \mathrm{~cm}^{2} / \mathrm{s}$, and a softness parameter $s=0.2$. In calculation of nuclei spectra with DRAGON, assuming a common injection index $\gamma_{i}=-2.32$ for all nucleons, this model reproduces the AMS-02 $\mathrm{B} / \mathrm{C}$ ratio [11] and proton spectrum [9] measurements, predicting a hardening in the proton spectrum without any break in the injection index, albeit too weak to be the sole explanation for the magnitude of index change as recently measured by CALET $[12,13]$ (an adaption of the model to CALET proton results is currently being investigated with the necessary changes expected not to have significant effect on the results of the study presented herein).

For the flux of the primary positron source causing the positron excess $\Phi_{e x}$, both pulsars and Dark Matter are considered, with a single young pulsar being the minimum solution assumed in the base model, for which the Monogem pulsar is chosen. The power-law with cut-off injection spectrum (defined by spectral index $\gamma_{e x}$ and cut-off energy $E_{c u t_{e x}}$ ) of each pulsar is propagated using the analytic solution of the propagation equation for a point source as explained e.g. in Ref. [14], adapted to include the gradual change in the diffusion coefficient, yielding the propagated flux from a pulsar as

$$
\Phi_{\text {pulsar }}=\frac{Q_{0} \eta}{\pi^{3 / 2} r_{d i f}^{3}} E^{-\gamma_{e x}}\left(1-\frac{E}{E_{\max }}\right)^{\left(\gamma_{e x}-2\right)} e^{-\frac{E / E_{\text {cutex }}}{1-E / E_{\max }}-\frac{r^{2}}{r_{\text {dif }}^{2}}},
$$

in which the characteristic diffusion distance $r_{d i f}$ is expressed as

$$
r_{d i f}=2 \sqrt{\frac{D(E) t_{d i f}}{1-\delta(E)} \frac{E_{\max }}{E}\left[1-\left(1-\frac{E}{E_{\max }}\right)^{(1-\delta(E))}\right]},
$$

with $E_{\max }=\left(b_{0} t_{\text {dif }}\right)^{-1}, b_{0}=1.410^{-16} \mathrm{GeV} \mathrm{s}^{-1}, D(E)$ given by Eq. 2.3 and $\delta(E)$ approximated as the local index of $D(E)$ at $E$.

The initial rotation energy of the pulsar $Q_{0}$ and the distance to the pulsar $r$ are taken from the ATNF catalog [15] for each pulsar, as well as its age. For the base model it is assumed that the emission of cosmic rays happens as a burst shortly after creation of the pulsar and the diffusion time $t_{\text {dif }}$ is therefore taken as the age of the pulsar.

The model is fitted to the data of CALET [4] based on total flux $\Phi_{e}^{-}+\Phi_{e}^{+}$and data of AMS-02 [5] for $E>10 \mathrm{GeV}$ based on $\Phi_{e}^{+}$by minimizing the sum of $\chi^{2}$ of both comparisons, with systematic uncertainties of both measurements taken into account. For the CALET measurement, the 1- $\sigma$ deviation $\Delta_{(k, i)}$ as a function of each data point's energy $\left(E_{i}\right)$ is listed in the supplemental material of Ref. [4] for the systematic uncertainty associated with the following parts of the analysis: Normalization, tracking, charge selection, electron identification, Monte Carlo. A systematic shift of the data-points is performed as part of the fit function with weights $w_{k}$ as free parameters and the 
squared weight of each uncertainty is added to the total $\chi^{2}$ of the fit as given by

$$
\chi_{\text {CALET }}^{2}=\left(\sum_{i} \frac{\left.\left(\Phi_{i}\right)+\sum_{k} \Delta_{(k, i)} w_{k}-J_{i}\right)}{\sigma_{i}^{2}}\right)+\sum_{k} w_{k}^{2},
$$

where $i$ iterates over the data points and $k$ over the different systematic uncertainty types. Systematic errors associated with the trigger and the boosted decision tree proton rejection are added quadratically to the statistical error. For the AMS-02 measurement, the error on mean energy $\sigma_{E}$ in each bin is translated into an error on flux $\sigma_{J(E)}$ using the power law index $\gamma_{e^{+}}$also shown in Ref. [5] via the relation $\sigma_{J(E)}=J(E)\left(\sigma_{E} / E\right)\left(\gamma_{e^{+}}-1\right)$.

For the study of Dark Matter signatures, the positron spectra (identical to electron spectrum due to the symmetry of the process) of Dark Matter annihilation or decay are calculated with PYTHIA 8.2 [16], and used as input for the propagation calculation with DRAGON, to obtain the flux at Earth. For the Dark Matter halo, a NFW profile with local density $\rho_{0}=0.3 \mathrm{GeV} / \mathrm{cm}^{3}$ is assumed.

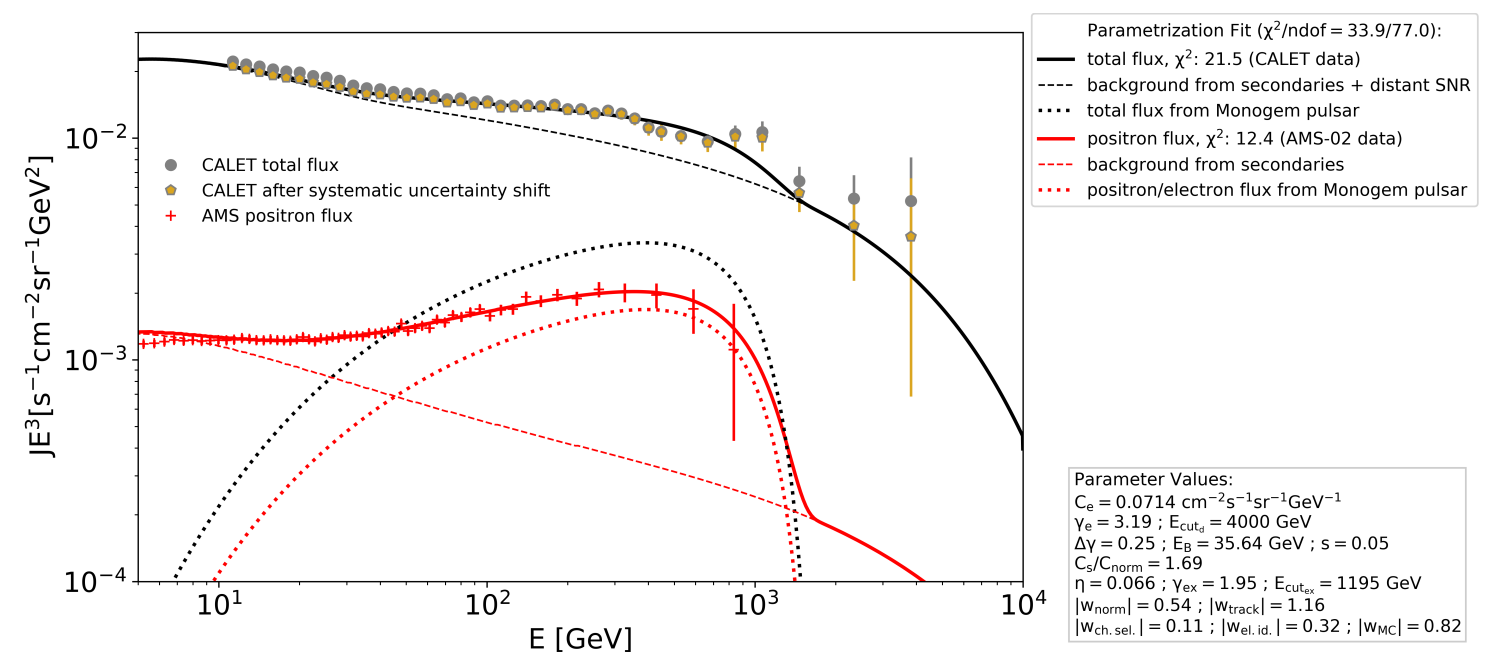

Figure 1: The base model as fitted to CALET [4] and AMS-02 [5] data. See legends for explanation of markers and lines, the values for the parameters introduced in the text are given in the box to the right of the graphs.

\section{Limits on Dark Matter Annihilation and Decay}

To obtain a limit on the speed averaged annihilation cross-section $\langle\sigma v\rangle$ or the lifetime $\tau$ of Dark Matter, the predicted flux from Dark Matter annihilation or decay is added to the base model as an additional component of $\Phi_{e x}$, with the scale factor increased in steps until $\chi^{2}$ reaches the 95\% CL threshold for the respective number of degrees of freedom (ndof). Multiplying (dividing) the normalization cross-section (lifetime) by the scale factor at which the $95 \% \mathrm{CL}$ threshold is crossed yields the limit on cross-section $\langle\sigma v\rangle$ (lifetime $\tau$ ). To avoid reporting a too stringent limit 
due to the fitting function having no unique minimum, the "Migrad" and "Simplex" minimizers of Minuit are used in alternation with different starting points as explained in Ref. [2]. By performing this procedure for various masses of the Dark Matter particles $\left(M_{D M}\right)$, limits as a function of $M_{D M}$ for various annihilation and decay channels are calculated, with those for leptonic channels shown in Fig. 2. The strictest limits are obtained for the channel of direct annihilation to electron+positron pairs, surpassing Fermi-LAT limits from $\gamma$-ray observation of dwarf galaxies below $200 \mathrm{GeV}$.
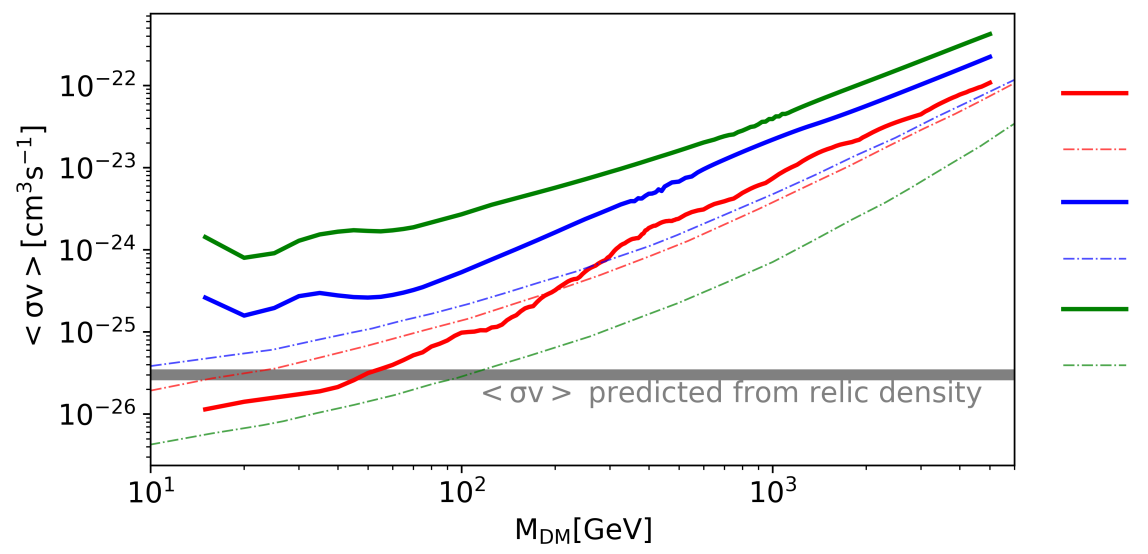

$\mathrm{DM}+\mathrm{DM} \rightarrow \mathrm{e}^{+}+\mathrm{e}^{-}$ Fermi-LAT dwarf galaxy limits

$\longrightarrow \mathrm{DM}+\mathrm{DM} \rightarrow \mu^{+}+\mu^{-}$ Fermi-LAT dwarf galaxy limits $\mathrm{DM}+\mathrm{DM} \rightarrow \tau^{+}+\tau^{-}$ Fermi-LAT dwarf galaxy limits

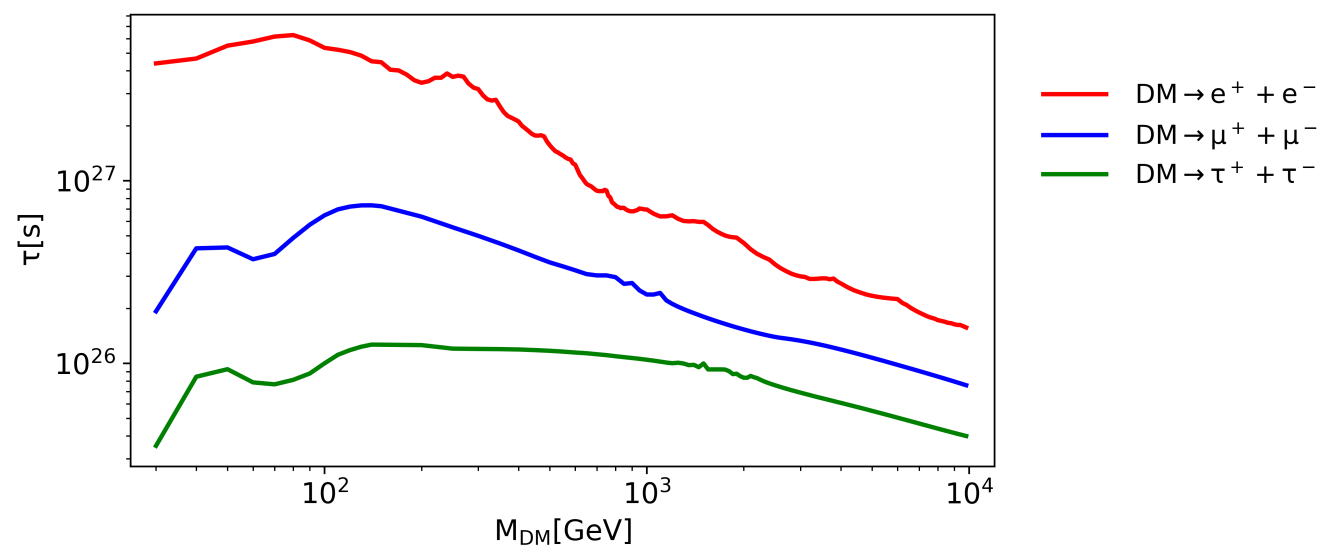

Figure 2: Limits on annihilation (top) and decay (bottom) as a function of $M_{D M}$ for the leptonic channels, compared to limits from $\gamma$-ray observation of dwarf galaxies with Fermi-LAT from the supplemental material of Ref. [17].

\section{Dark Matter Interpretation of Structures}

As described above, the addition of the predicted dark matter flux and increase of the scale factor for limit calculation causes eventually an increase in $\chi^{2}$ of the fit. However, it is found that for annihilation/decay directly into electron+positon pairs, the addition of the Dark Matter flux with a scale factor smaller than the limit value improves the fit singificantly compared to the base model with a pulsar extra source in two ranges of $M_{D M}$, corresponding to the step-like structures near $350 \mathrm{GeV}$ and $1 \mathrm{TeV}$ in the CALET spectrum. 
As a speculative interpretation of the structures by Dark Matter annihilation (decay), the optimal cross-section (lifetime) for modeling the structures and the associated maximal $\chi^{2}$ reduction are determined depending on $M_{D M}$ with the results shown in Fig.3. The largest $\chi^{2}$ improvement compared to the single pulsar case is $\Delta \chi^{2}=6.64$ for $M_{D M}=350 \mathrm{GeV}$ in the annihilation case as shown in Fig.4, and $700 \mathrm{GeV}$ for decay, exceeding the 95\% significance level for two additional free parameters $\left(M_{D M}\right.$ and $<\sigma v>$ or $\tau$ respectively).
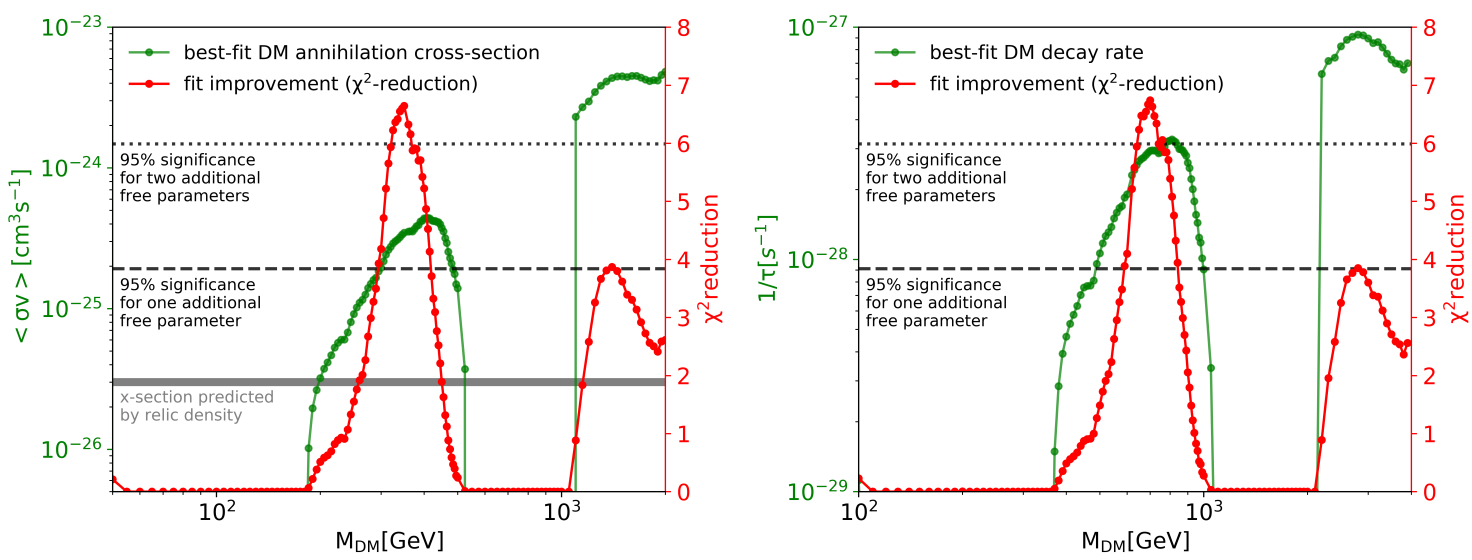

Figure 3: Fit improvement by addition of flux from Dark Matter to the base model for annihilation (left) and decay (right) as a function of $M_{D M}$, together with the annihilation cross-section or inverse lifetime giving the best fit.

\section{Multi-Pulsar Interpretation of the $350 \mathrm{GeV}$ Structure}

The fit improvement by addition of Dark Matter indicates that the structure near $350 \mathrm{GeV}$ has significance within the modeling framework used in this study, warranting an explanation. As a more mundane alternative to Dark Matter, a refinement of the astrophysical background model was investigated. While the release of high energy electrons and positrons from the Monogem pulsar coincides with its creation in the base model, it is expected that they are confined in the PWN initially and released with the disruption of the PWN, introducing the release delay $T_{r}$ as another parameter for which the range up to $100 \mathrm{kyr}$ is considered [18]. In addition, while the single pulsar model fits the current data well, it is natural to assume that multiple nearby pulsars contribute to the source of the positron excess, based on the observations summarized in the ATNF catalog [15]. Relevant candidates were selected from its entries with age less than $1 \mathrm{Myr}$ and distance less than $1 \mathrm{kpc}$ by their potential contribution to the total flux being more than $5 \%$ for any combination of $\gamma_{e x}$ and $E_{c u t_{e x}}$, resulting in 13 remaining candidates. The refined model with the combined flux from these 13 pulsars as $\Phi_{e x}$ was fitted to the data keeping $\eta, \gamma_{e x}$ and $E_{c u t_{e x}}$ as free parameters common for all pulsars, and scanning over $T_{r}$ and $E_{\text {cut }_{d}}$ in steps. A minimum value of $\chi^{2}=26.2$ is found, with marginal differences for several cases with $T_{r}>=60 \mathrm{kyr}$. The case of $T_{r}=60 \mathrm{kyr}$ is shown in Figure 5 , demonstrating that the $350 \mathrm{GeV}$ structure is represented well by this multi-pulsar model. 


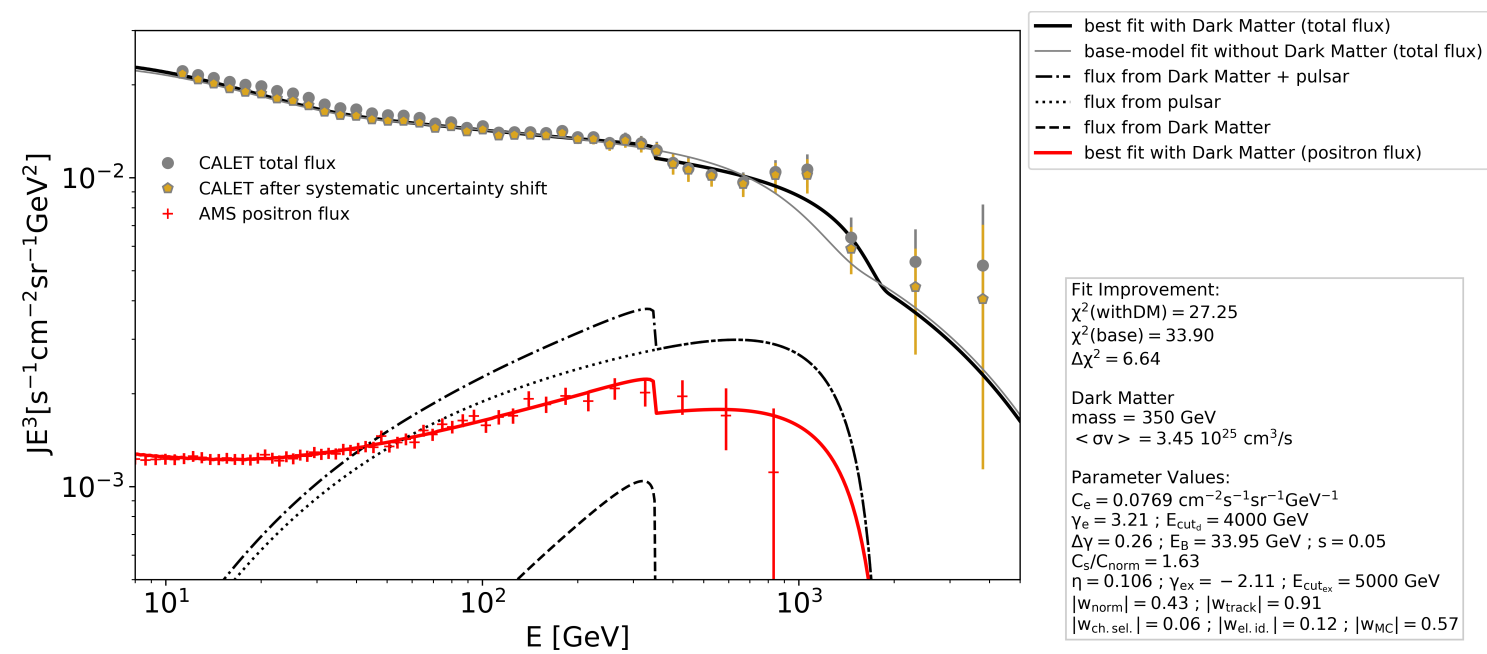

Figure 4: Model adding the flux from direct annihilation of $350 \mathrm{GeV}$ Dark Matter into electronpositron pairs to the base model, improving the fit quality by $\Delta \chi^{2}=6.64$. See legend for explanation of markers and lines, parameter values are given in the box to the right of the graph.

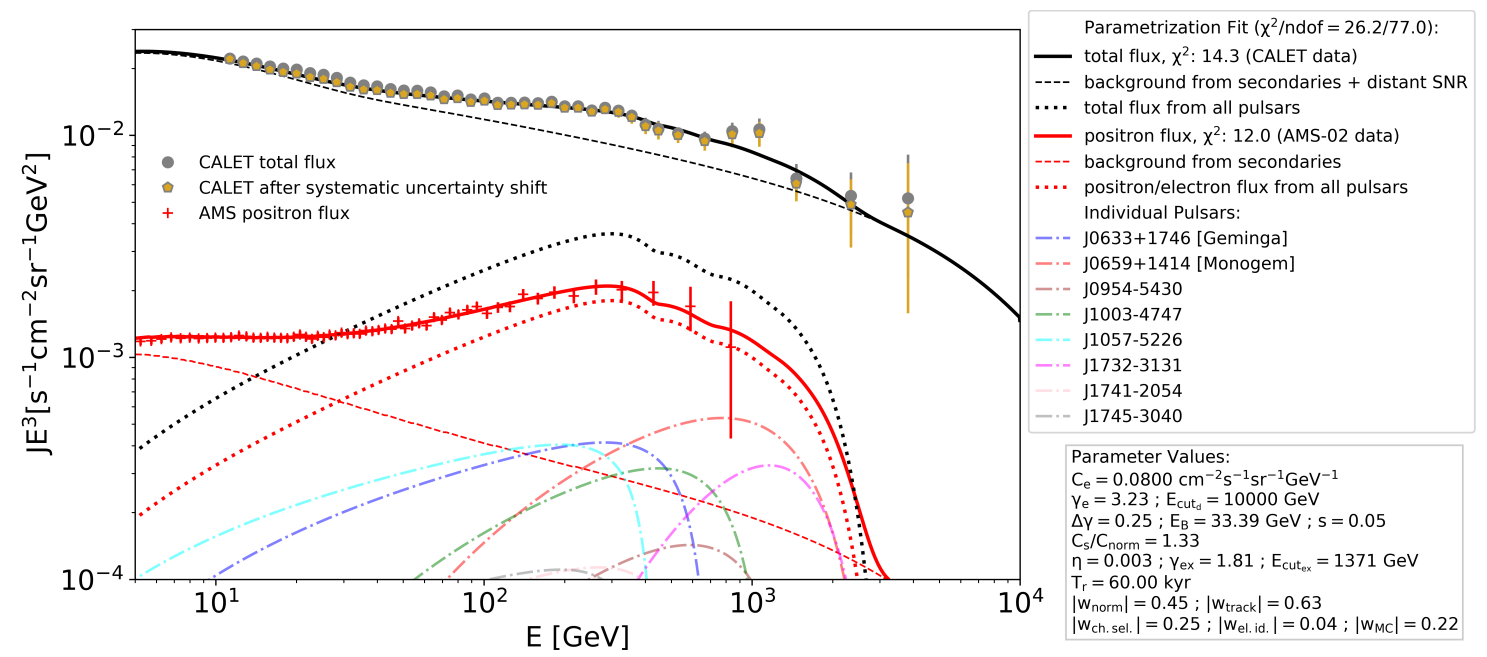

Figure 5: The multi-pulsar model with $T_{r}=60 \mathrm{kyr}$. See the legend for explanation of markers and lines, parameter values are given in the box to the right of the graph. Only those of the 13 extra-source pulsars which have high enough flux to appear in the plot are listed in the legend. 


\section{Summary and Outlook}

Limits on annihilation of Dark Matter with mass up to $5 \mathrm{TeV}$ and decay of Dark Matter with mass up to $10 \mathrm{TeV}$ have been calculated from from the CALET electron+positron spectrum in combination with AMS-02 data. These limits give strong constraints not subject to halo model uncertainties except for the local Dark Matter density, especially on the direct electron+positron annihilation (decay) channel in which least $\gamma$-rays are produced. The results emphasize the complementarity between indirect Dark Matter searches in electron/positron cosmic rays and $\gamma$-rays.

A step-like structure appearing in the CALET spectrum at $350 \mathrm{GeV}$ is shown to be compatible with an interpretation as a Dark Matter signature or an explanation by astrophysical origin. In the latter case it is shown to be a possible hint for the presence of individual local astrophysical sources, with the model combining the flux of multiple nearby pulsars as the source of the positron excess showing a significant fit improvement over the single-pulsar base model.

Reduction of systematic errors and better understanding of their energy dependence is expected to further increase the precision of the CALET measurement in the future, which together with the ongoing extension of statistics and the upper energy bound [19] should improve the limits and possibly the significance of the spectral structures.

\section{References}

[1] Y. Asaoka, et al., PoS ICRC2019 (2019). In press.

[2] H. Motz, Y. Asaoka, S. Torii, S. Bhattacharyya, JCAP 1512, 047 (2015).

[3] N. Hiroshima, M. Hayashida, K. Kohri, Phys. Rev. D99, 123017 (2019).

[4] O. Adriani, et al., Phys. Rev. Lett. 120, 261102 (2018).

[5] M. Aguilar, et al., Phys. Rev. Lett. 122, 041102 (2019).

[6] O. Adriani, et al., Phys. Rev. Lett. 119, 181101 (2017).

[7] G. Ambrosi, et al., Nature 552, 63 (2017).

[8] D. Gaggero, L. Maccione, G. Di Bernardo, C. Evoli, D. Grasso, Phys.Rev.Lett. 111, 021102 (2013).

[9] M. Aguilar, et al., Phys. Rev. Lett. 114, 171103 (2015).

[10] H. Motz, Y. Asaoka, S. Torii, S. Bhattacharyya, PoS ICRC2017, 265 (2018).

[11] M. Aguilar, et al., Phys. Rev. Lett. 117, 231102 (2016).

[12] O. Adriani, et al., Phys. Rev. Lett. 122, 181102 (2019).

[13] P. Marrocchesi, et al., PoS ICRC2019 (2019). In press.

[14] J. Feng, H.-H. Zhang, The European Physical Journal C 76, 229 (2016).

[15] R. N. Manchester, G. B. Hobbs, A. Teoh, M. Hobbs, Astron.J. 129, 1993 (2005).

[16] T. Sjöstrand, et al., Computer Physics Communications 191, 159 (2015).

[17] M. Ackermann, et al., Phys. Rev. Lett. 115, 231301 (2015).

[18] D. Malyshev, I. Cholis, J. Gelfand, Phys.Rev. D80, 063005 (2009).

[19] S. Torii, et al., PoS ICRC2019 (2019). In press. 